\title{
A rapid passage through a two-active-X-chromosome state accompanies the switch of imprinted X-inactivation patterns in mouse trophoblast stem cells
}

\author{
Julie Prudhomme ${ }^{1}$, Agnès Dubois ${ }^{1,2}$, Pablo Navarro ${ }^{2}$, Danielle Arnaud ${ }^{1}$, Philip Avner ${ }^{1,3}$ and Céline Morey ${ }^{1,4^{*}}$
}

\begin{abstract}
Background: In female mice, while the presence of two-active $X$-chromosomes characterises pluripotency, it is not tolerated in most other cellular contexts. In particular, in the trophoblastic lineage, impairment of paternal $X\left(X^{P}\right)$ inactivation results in placental defects.

Results: Here, we show that Trophoblast Stem (TS) cells can undergo a complete reversal of imprinted X-inactivation without detectable change in cell-type identity. This reversal occurs through a reactivation of the $X^{P}$ leading to TS clones showing two active $X$ s. Intriguingly, within such clones, all the cells rapidly and homogeneously either re-inactivate the $X^{P}$ or inactivate, de novo, the $X^{M}$.

Conclusion: This secondary non-random inactivation suggests that the two-active-X states in TS and in pluripotent contexts are epigenetically distinct. These observations also reveal a pronounced plasticity of the TS epigenome allowing TS cells to dramatically and accurately reprogram gene expression profiles. This plasticity may serve as a back-up system when $\mathrm{X}$-linked mono-allelic gene expression is perturbed.
\end{abstract}

Keywords: Imprinted X-inactivation, Trophoblast Stem cells, Epigenetic Reprogramming

\section{Background}

In male mammals, the $\mathrm{XY}$ genotype results in the monoallelic expression of X-linked genes. In contrast, in females, the presence of two X-chromosomes may lead to bi-allelic X-linked expression, which is known to be detrimental to the embryo [1]. To prevent this double dose of X-linked products, female mammals inactivate one $\mathrm{X}$-chromosome. In the mouse, two forms of X-inactivation exist. A completely biased inactivation of the $\mathrm{X}^{\mathrm{P}}$ is first established in the 4-cell embryo [2-4]. At the morula-blastocyst transition, the $\mathrm{X}^{\mathrm{P}}$ is reactivated in cells of the inner cell mass (ICM)-one of the two cell types known to be able to withstand two active Xs-while the inactivation of the $\mathrm{X}^{\mathrm{P}}$ persists in the extraembryonic

\footnotetext{
*Correspondence: celine.morey@univ-paris-diderot.fr

${ }^{4}$ CNRS, UMR7216 Epigenetics and Cell Fate, 35 rue Hélène Brion,

75013 Paris, France

Full list of author information is available at the end of the article
}

lineages of the trophectoderm and of the primitive endoderm and in their placental and yolk sac derivatives, respectively [3, 5-7]. As opposed to this imprinted inactivation (I-XCI), upon epiblast formation, ICM cells independently choose to inactivate either the $\mathrm{X}^{\mathrm{P}}$ or the maternal $\mathrm{X}\left(\mathrm{X}^{\mathrm{M}}\right)$ at random [8]. This initial choice is then clonally inherited thereby giving rise to an allelic mosaicism of X-linked gene expression within female adult tissues. This random X-inactivation ( $\mathrm{R}-\mathrm{XCI})$ is extremely stable and is only reverted in germ cells, which, therefore, constitute the second cell type known to lack XCI.

Both I-XCI and R-XCI rely on the same basic mechanism: the overexpression of the Xist gene from the future inactive $\mathrm{X}$ and a cis-accumulation of Xist ncRNAs, which triggers a cascade of epigenetic changes ending up in the formation of a heterochromatic X-chromosome (for review see $[9,10])$. Beyond this common core mechanism, lineage-specific differences in the establishment 
and stability of the inactive state have been investigated in vivo, during the blastocyst development, but also ex vivo, using cellular models of the three blastocyst lineages, namely the embryonic stem (ES) cells [11], the trophoblast stem (TS) cells [12] and the extraembryonic endoderm stem (XEN) cells [13]. Intriguingly, amongst these different cell types, there seems to be a correspondence between the cell potency, the degree of stability of the inactive state and the level of tolerance of X-linked bi-allelic expression. Pluripotent ES cells stand at the extremity of this continuum since they relatively happily maintain two active Xs. A control of X-inactivation initiation by pluripotency markers and, reciprocally, a stabilisation of the naïve pluripotent state by two active $\mathrm{X}$-chromosomes have been suggested to sustain this equilibrium $[14,15]$.

In contrast, the multipotent trophoblast cells appear especially refractory to any global deregulation of $\mathrm{X}$-chromosome expression since bi-allelic X-linked gene expression in the trophectoderm of embryos carrying mutations in paternal alleles of Xist results in lethality due to extraembryonic defects [16, 17]. Paradoxically, this latter lineage is particularly rich in gene escaping from I-XCI-i.e. genes expressed from both Xs-compared to other adult cell types $[18,19]$. In addition to this, transient and spontaneous reactivations of certain $\mathrm{X}$-linked genes occur both and ex vivo [20] and, after differentiation, the relaxation of I-XCI extends to additional genes in specific subtypes of placental cells [21-25]. Even more dramatically, complete inversion of XCI profiles has been observed in few spongiotrophoblast progenitor cells before the appearance of global placental defects in embryos carrying a paternal Xist mutation [26]. Since the $\mathrm{X}$-chromosome is enriched in genes involved in placental functions compared to most autosomes [27], these observations suggest that the trophectoderm is the site of an opposition between the requirement for X-chromosome plasticity of expression necessary to commit into multiple trophoblastic fates and the need to maintain specific $\mathrm{X}$-linked genes under a tight dosage control in specific subtypes of placental cells to ensure that the placenta functions properly.

In order to understand how the plasticity of X-chromosome expression is regulated in the trophectoderm lineage, we used TS cells carrying a mutation in the maternal $\mathrm{X}$-linked gene Hprt1. In this context, the expression of Hprt1 serves as an index of X-chromosome activity and cells that re-express the unmutated, paternal, Hprt1 copy can be selected for with aminopterin (HAT medium). Using this system, we isolated, among others, HAT-resistant clones showing a reversal of I-XCI profiles characterised by an inactive $\mathrm{X}^{\mathrm{M}}$ and a reactivated $\mathrm{X}^{\mathrm{P}}$. This reversal is mediated by a passage through a
two-active-X state and followed by a de novo inactivation of the $\mathrm{X}^{\mathrm{M}}$ involving an accumulation of Xist RNAs on the chromosome and a recruitment of H3K27me3 silent histone mark at most-but not all-X-linked genes. Importantly, within clonal cell populations showing two-active $\mathrm{X}$-chromosomes, all cells homogeneously choose to inactivate the same chromosome: the $\mathrm{X}^{\mathrm{P}}$ in most clones, or the $\mathrm{X}^{\mathrm{M}}$ in rare instances. No mosaic clones constituted of a mixture of cells with an inactive $\mathrm{X}^{\mathrm{M}}$ and of cells with an inactive $\mathrm{X}^{\mathrm{P}}$ have been observed. This suggests that the choice process used, in TS cells, after the initial reactivation of the $\mathrm{X}^{\mathrm{P}}$ is different from a random $\mathrm{X}$-chromosome choice as it is described to occur upon differentiation of epiblast cells or upon differentiation of ES cells. This furthermore indicates that the two-active-X state in TS cells is epigenetically different from the two-active- $\mathrm{X}$ state in pluripotent cells.

\section{Results}

\section{Cells expressing the Hprt1 gene from the paternal} $\mathrm{X}$-chromosome pre-exist in undifferentiated populations of female TS cells

In order to evaluate the stability of I-XCI in undifferentiated TS cells, we used a female TS cell line (F3) carrying a mutation at the X-linked Hprt locus $\left(\mathrm{Hprt}^{\mathrm{bml}}\right)$ associated with a retroviral insertion in the maternal Hprt1 gene, which leads to a stable loss-of-function of the maternal allele [28]. In such a genetic configuration, the expression of the Hprt1 locus can be theoretically used as an index of $\mathrm{X}^{\mathrm{P}}$ and $\mathrm{X}^{\mathrm{M}}$ activities by growing cells on two different selective media. Growth on medium complemented with HAT (Hypoxanthine Aminopterin Thymidine) selects for cells expressing a functional, paternal, $H p r t 1^{W T}$ allele, while only cells unable to use the regular HPRT1 pathway, which have, therefore, supposedly, inactivated the paternal $\mathrm{Hprt1}^{\text {WT }}$ copy, will survive on 6-TG (6-ThioGuanine) medium. In addition, the F3 cell line carries an $\mathrm{X}^{\mathrm{M}}$ of 129Sv.Pgk1a origin and an $\mathrm{X}^{\mathrm{P}}$ of $129 \mathrm{~Sv}$ origin allowing the identification of allele origin through the extensive polymorphisms located in the large Pgk1a-derived region surrounding the Xist gene.

We first determined the frequency of spontaneous reversal of the [Hprt-] phenotype (previously estimated to be $<10^{-8}$ in primary fibroblasts [28]) by growing $\mathrm{Hprt}^{\mathrm{bm1}}$ male TS cells (F2 cell line) on HAT medium (Fig. 1a, b). No HAT-resistant clones were observed in the time course of three independent experiments (experiments $\# A, \# B$ and $\# C$ ), indicating that the rate of [Hprt-] spontaneous reversal is very low in TS cells and will not significantly influence our results (Fig. 1b). Similarly, the vast majority of F3 female TS cells died upon HAT selection confirming that the paternal $H p r t 1^{W T}$ allele is silenced by $\mathrm{I}-\mathrm{XCI}$ in most cells. A number of HAT-resistant F3 clones 


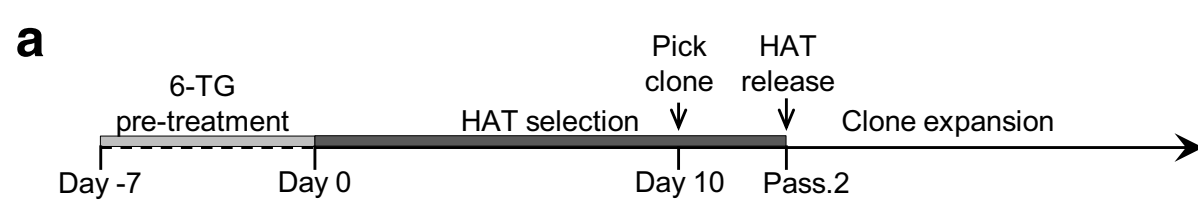

\begin{tabular}{|c|c|c|c|c|c|c|c|c|}
\hline \multirow[t]{3}{*}{ b } & & \multicolumn{6}{|c|}{ Experiment \# } & \multirow{3}{*}{$\begin{array}{l}\text { Average } \\
\text { frequency }\end{array}$} \\
\hline & & A & B & $\mathrm{C}^{*}$ & $\mathrm{D}$ & $E$ & $\mathrm{~F}$ & \\
\hline & $\mathrm{Nb}$ of plated cells & $2.10^{6}$ & $2.10^{6}$ & $2.10^{6}$ & $2.10^{6}$ & $4.10^{6}$ & $4.10^{6}$ & \\
\hline \multirow{3}{*}{$\begin{array}{c}\text { Number of } \\
\text { HAT resistant } \\
\text { clones }\end{array}$} & $\begin{array}{c}\text { XX TS (F3) } \\
\text { direct HAT treatment }\end{array}$ & 18 & 21 & 26 & ND & 54 & 48 & $\begin{array}{c}1,15.10^{-5} \\
\left( \pm 0,18 \cdot 10^{-5}\right) \\
\end{array}$ \\
\hline & $\begin{array}{c}\mathrm{XX} \text { TS }(\mathrm{F} 3) \\
\text { pre-treated with 6-TG }\end{array}$ & 8 & 14 & 15 & 19 & ND & ND & $\begin{array}{c}0,7 \cdot 10^{-5} \\
\left( \pm 0,2 \cdot 10^{-5}\right)\end{array}$ \\
\hline & $\begin{array}{c}\text { XY TS (F2) } \\
\text { direct HAT treatment }\end{array}$ & 0 & 0 & 0 & ND & ND & ND & 0 \\
\hline
\end{tabular}

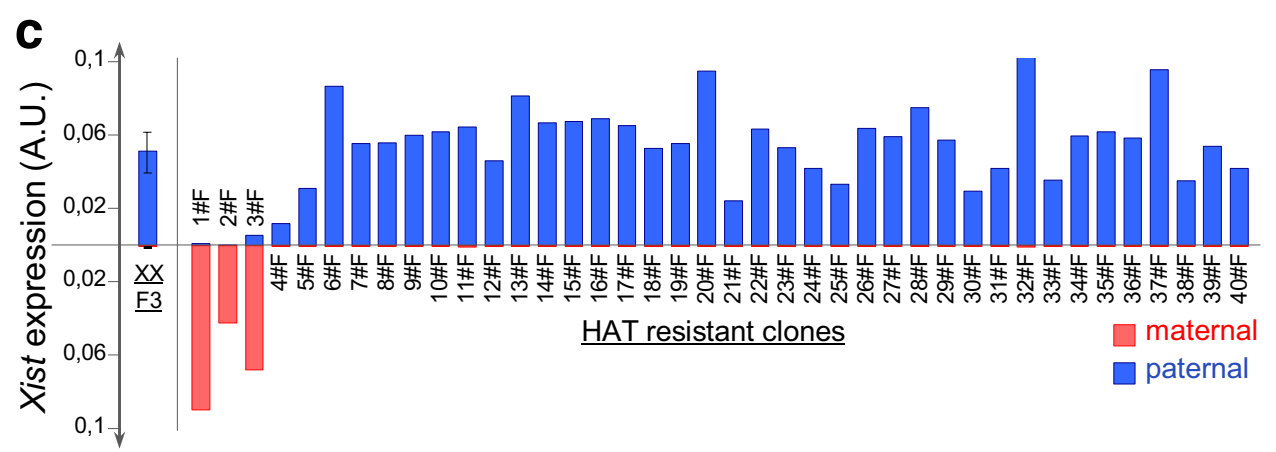

Fig. 1 HAT-resistant clones of F3 female TS cells show various patterns of Xist expression including an inverted Xist expression profile. a Protocol of isolation of HAT-resistant TS clones. Female F3 TS cells were either directly treated with HAT or pre-treated with 6-TG before HAT selection. HATresistant clones were picked at day 10 of selection and maintained under HAT selection for 2 additional passages. Analysis of Xist expression was performed after 8 passages. b Table showing the frequencies of HAT-resistant clones obtained in the time course of 6 independent experiments (\#A, $\# B, \# C, \# D, \# E$ and $\# F)$. The frequency of HAT-resistant clones after pre-treatment with 6-TG is significantly different from the frequency of HAT-resistant clones in the absence of 6-TG pre-treatment (Mann-Whitney test, $p$ value $<0.05$ ). The asterisk indicates that in experiment \#C, the HAT selection has been maintained for a longer period of time, see Additional file 5 A. The protocol described in panel $\mathbf{a}$ has been applied in the other experiments (i.e. $\# A, \# B, \# D, \# E$, and $\# F$ ). In this study, each individual clone analysed is identified by a capital letter which refers to the experiment from which the clone has been isolated and by a number which identifies each clone of a given experiment. Clones that have been characterised further in this study all come from HAT selection without 6-TG pre-treatment. c Cumulative histograms showing the Xist expression levels measured by allelic RT-qPCR after standardisation by the Rplp0 reporter gene [20] in the 40 clones obtained in experiment \#F (passage 8 after clone picking). Paternal and maternal expressions are depicted in blue and red above and below the $x$-axis, respectively. For F3 parental female cells, mean and standard deviation have been calculated on the basis of three independent cell cultures. A.U. Arbitrary Units

were, however, observed with a consistent frequency of $\sim 10^{-5}$ over five independent experiments (experiments \#A, \#B, \#C, \#E and \#F; Fig. 1b). This suggests that some female F3 TS cells have reactivated the paternal Hprt ${ }^{W T}$ allele.

In order to rule out the possibilities that these HATresistant clones were all descendants of rare events that occurred at the time of F3 cell line derivation or that the HAT treatment, by itself, triggers the paternal $\mathrm{Hprt}_{1}{ }^{W T}$ reactivation, we first sub-cloned the F3 cell line and tested four independent sub-clones for their ability to generate HAT-resistant cells. Significant frequencies of
[Hprt-] reversal were obtained with all four sub-clones indicating that each F3 TS cell has a similar probability and ability to reactivate the paternal $H p r t 1^{W T}$ allele (data not shown). Secondly, we grew F3 TS cells on 6-TG prior to HAT selection in order to eliminate potential pre-existing $[\mathrm{Hprt}+]$ cells from the population before HAT selection. Under these conditions, we still recovered HAT-resistant clones with a significant frequency $\left(\sim 0.7 \times 10^{-5}\right)$ compared to spontaneous [Hprt-] reversal in F2 male cells again confirming that F3 TS cells are homogeneously able to induce $H p r t 1^{W T}$ reactivation (experiments \#A, \#B, \#C and \#D; Fig. 1b). This frequency, 
however, was significantly and reproducibly lower than the frequency obtained upon direct HAT selection suggesting that TS cells displaying an active $H_{p r t 1} 1^{W T}$ paternal allele are naturally present amongst the F3 cell population.

Importantly, HAT-resistant clones appeared morphologically indistinguishable from parental F3 cells (see Additional file 1A). In addition, when we analysed the expression of classical TS cell markers and markers specific for differentiated TS states in HAT-resistant cells, most clones showed expression profiles similar to undifferentiated F3 cells or to the other TS cell lines we tested, indicating that these HAT-resistant cells displayed the characteristics of bona fide TS cells (see Additional file 1B). We concluded from these observations that female TS cells are prone to spontaneously reactivate the Hprt $1^{W T}$ locus on the paternal X-chromosome and recurrently do so at a low but significant frequency during cell growth.

\section{Reactivation of the paternal Hprt 1 locus is accompanied by a switch of $X$-inactivation profiles in a subset of HAT-resistant TS cells}

In order to determine the extent of $\mathrm{X}^{\mathrm{P}}$ reactivation amongst various HAT-resistant clones and, in particular, establish the frequency of clones that may have reactivated the entire $\mathrm{X}^{\mathrm{P}}$, we analysed the expression of Xist using allelic RT-qPCR in all the clones recovered during the time course of a single HAT selection (experiment $\#$ F). Strikingly, we observed two distinct Xist expression profiles: 37 out of 40 clones displayed paternal Xist expression levels similar to parental F3 cells, while 3 clones showed a maternally restricted expression of Xist (Fig. 1c). When we performed Xist RNA-FISH on representative clones of each category, we observed a single Xist domain in most nuclei of both categories (see Additional file 2A). This suggests that the clones expressing Xist from the $\mathrm{X}^{\mathrm{P}}$ locally reactivated the paternal Hprt1 ${ }^{\text {WT }}$ gene. This is confirmed by the transcription of the Hprt $1^{W T}$ alleles from the Xist-coated X-chromosome in a significant percentage of cells of these clones (as determined by RNA-FISH) and by a preferential expression of maternal alleles of other X-linked genes as assessed both by allelic RT-qPCR and by RNA-FISH (see Additional file $2 \mathrm{~B}, \mathrm{C}$ ). We noticed variability in the percentage of cells transcribing the Hprt $1^{W T}$ allele between clones (see Additional file 2C). Since the analysis has been performed several passages after HAT release, this suggests that the $H p r t 1^{W T}$ allele has been silenced de novo in a percentage of cells that varies from clone to clone. Similar transient and local reactivations of Hprt1 and of several X-linked loci on the inactive $\mathrm{X}^{\mathrm{P}}$ have been previously reported to occur spontaneously and at low frequency in another TS cell line and in the cells of the Trophectoderm in vivo [20].

We, therefore, focussed our study on the minority of clones in which the reactivation of the paternal Hprt $1^{\text {WT }}$ allele is accompanied by a loss of paternal Xist expression and by an induction of maternal Xist RNA accumulation, which suggests that these TS cells have undergone a complete reversal of the Xist expression profile. To verify this hypothesis, we first analysed, by allelic RT-qPCR, the expression of eight X-linked genes located within the $129 \mathrm{~Sv} /$ Pgk1a polymorphic region and of Hprt1, in eight Xist revertant clones collected from independent HAT selections (Fig. 2a and see Additional file 2D; each individual clone is identified by a capital letter which refers to the experiment from which the clone has been isolated and by a number which identifies each clones within a given experiment). Most X-linked genes-except for Pbdc1 (also called 2610029G23Rik), which is known to escape from X-inactivation in most tissues [29]appeared to be preferentially expressed from the $X^{P}$. We noted however that some $\mathrm{X}$-linked genes including $P g k 1, C o x 7 b$ and Atp $7 a$ showed variable levels of residual expression from the $\mathrm{X}^{\mathrm{M}}$ in these revertants. This suggests that a subset of cells within these clones still express the maternal alleles of these genes at a low level.

In parallel with these quantifications, we performed Xist RNA-FISH in combination with Hprt1 (which identifies transcription from the $\mathrm{X}^{\mathrm{P}}$ ) and 7 other $\mathrm{X}$-linked genes (including loci located outside of the $129 \mathrm{~Sv} /$ Pgk1a polymorphic region) (Fig. 2b, c). In the three Xist revertant clones that were analysed, we observed a preferential mono-allelic expression from the active $\mathrm{X}$ for Hprt1 and the other X-linked genes that were tested. This confirms that the $\mathrm{X}^{\mathrm{P}}$ has been reactivated, while the $\mathrm{X}^{\mathrm{M}}$ has been inactivated de novo in these clones. As expected, $K d m 5 c$, an X-inactivation escaper [29], was expressed from both Xs.

Thus, Xist inverted expression profile is associated with a chromosome-wide allelic switch of X-inactivation patterns in the majority of cells of a subset of HAT-resistant TS cell clones.

\section{De novo X-inactivation of the maternal X-chromosome is associated with an enrichment of $\mathrm{H} 3 \mathrm{~K} 27 \mathrm{me} 3$ at most, but not all, $X$-linked genes}

In order to test whether this de novo silencing of the $\mathrm{X}^{\mathrm{M}}$ in I-XCI revertant clones is accompanied by the chromatin changes associated with $\mathrm{X}$-inactivation, we analysed the distribution of H3K27me3, a silent histone mark known to be recruited at the inactive $X$ [30-32], using immunofluorescence against this histone mark combined with RNA-FISH for Xist. As in F3 parental TS cells, in two different revertant clones, the Xist domain 

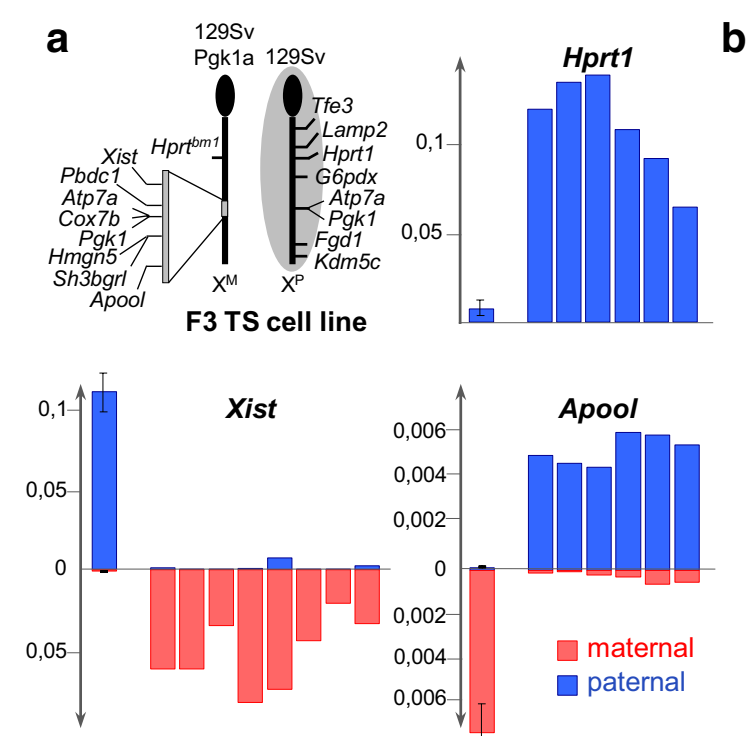

b
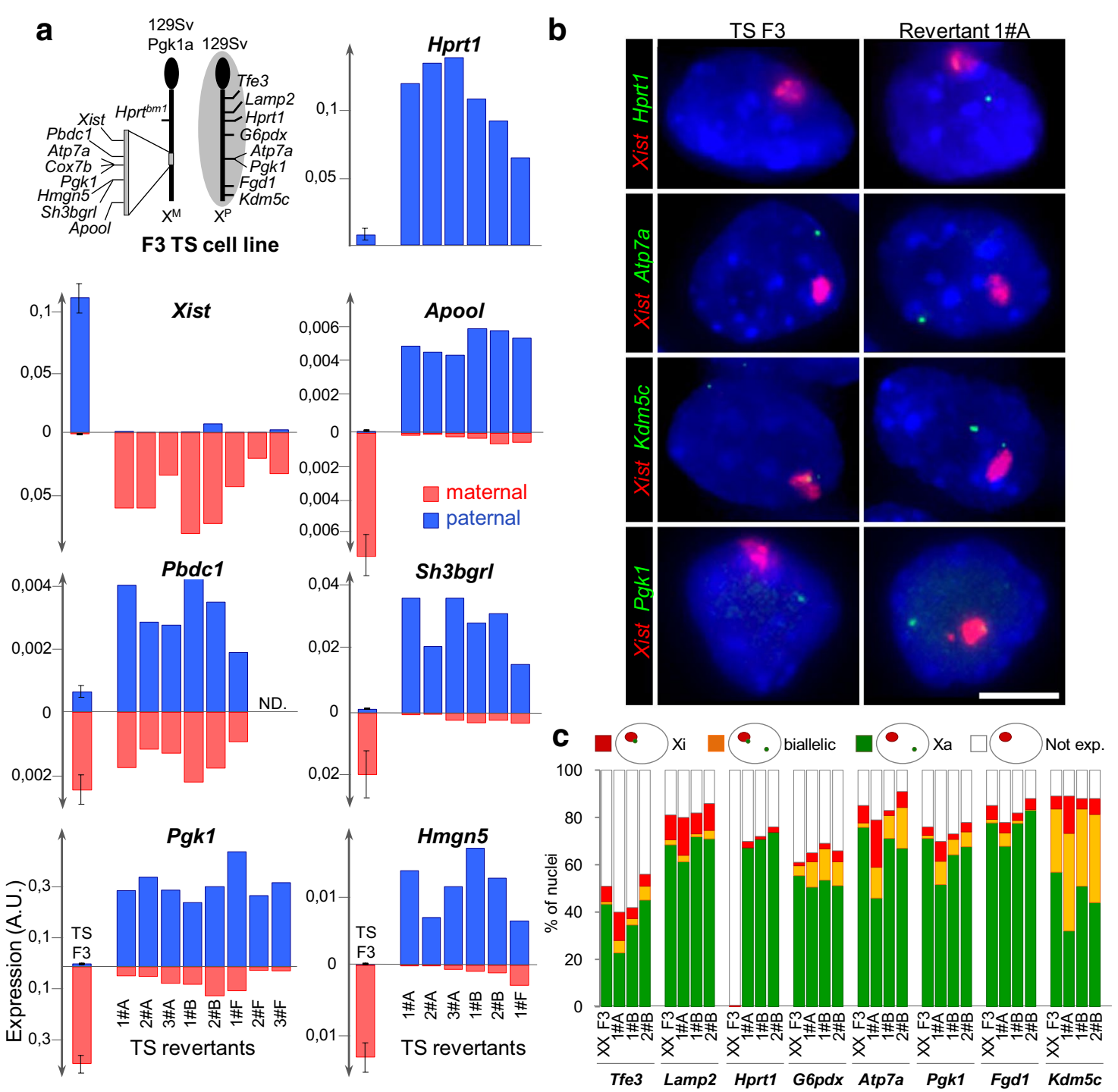

Fig. 2 Allelic inversion of Xist expression is associated with a reversal of X-inactivation profiles. a Cumulative histograms showing the expression levels of several X-linked genes located within the 129/Pgk1 a polymorphic region measured by allelic RT-qPCR in the 8 Xist revertant clones from independent HAT selection experiments (analysis performed at passage 8 or later after clone picking). Standardisation by Rplp0 has been applied. Paternal and maternal expressions are depicted in blue and red above and below the $x$-axis, respectively. The diagram shows the genomic positions of the genes analysed by RT-qPCR (on the 129/Pgk1a chromosome) and by RNA-FISH (on the 129 chromosome) in pane/ a and b. For F3 parental female cells, mean and standard deviation have been calculated on the basis of three independent cell cultures. A.U. Arbitrary Units. b Representative images of double RNA-FISH for Xist and for the indicated X-linked gene in F3 parental cells and in cells of clone 1\#A. The inactive X-chromosome is identified through Xist RNA accumulation (red). Primary transcription at the indicated X-linked gene is co-detected in green. $<1 \%$ of F3 parental TS cells show an Hprt1 signal in agreement with the inactivation of the wild-type, paternal, Hprt1 allele. In contrast, an Hprt1 signal away from the Xist domain is detected in the majority of nuclei of clone $1 \# A$ indicating a reactivation of the $X^{P}$ carrying the wild-type Hprt1 gene. Scale $b a r=5 \mu \mathrm{m}$. c Cumulative histograms of the percentages of nuclei with the depicted expression pattern in F3 TS cells and in nuclei of 3 independent Xist revertant clones (analysis performed at passage 8 or later after clone picking). See Additional file 10 for probe position. No significant difference is observed between any of the clones and F3 parental cells for all X-linked genes except for Hprt1 ( $X^{2}$ test $p$ value $\left.<0.05\right)$. $n>150$

appeared consistently associated with an accumulation of H3K27me3 (see Additional file 3A, B).

ChIP-chip analyses for H3K27me3 in male TS cells (F2 cell line) and in F3 and revertant clone 1\#A female TS cells further specified that this histone mark preferentially associated with alleles on the inactive $\mathrm{X}$ that are expressed from the active $\mathrm{X}$ in TS cells as compared to intergenic regions or to X-linked genes that are silent in TS cells, as previously described in other cell types showing either R-XCI or I-XCI $[20,30]$ (see 


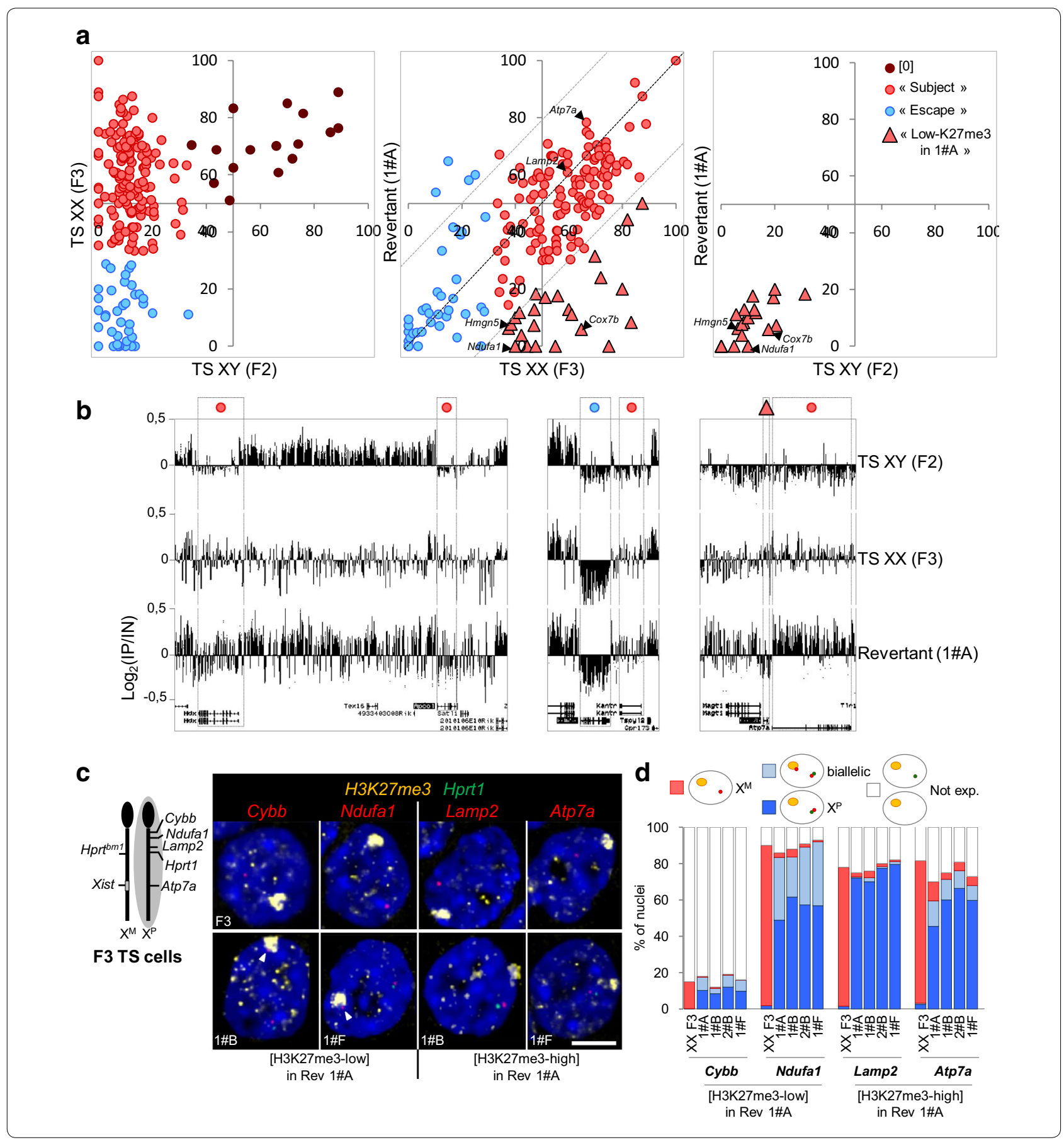

Additional file 3C, D, E). The level of H3K27me3 at expressed X-linked genes in I-XCI revertant 1 \#A was however significantly lower than H3K27me3 levels in the F3 parental cell line. Gene-by-gene clustering of H3K27me3 profiles in F2, F3 and clone 1\#A revealed that this loss of $\mathrm{H} 3 \mathrm{~K} 27 \mathrm{me} 3$ in 1 \#A cells compared to female F3 cells only occurs at $12 \%$ of expressed genes analysed, leading, at these genes, to an H3K27me3 percentage very similar to that observed in male F2 cells (Fig. 3a, b and see Additional file 4).

We then addressed whether the level of H3K27me3 in the gene bodies impacted gene transcription, using immuno-RNA-FISH. To specifically distinguish the $\mathrm{X}^{\mathrm{P}}$, we analysed Hprt1 transcription, whilst the transcription and the topological localisation of $4 \mathrm{X}$-linked loci (Cybb, Ndufa1, Lamp2 and Atp7a) were compared to the 
(See figure on previous page.)

Fig. 3 Xist RNA domain on the maternal inactive X in I-XCl revertant clones is associated with and accumulation of H3K27me3 at the majority of expressed X-linked genes. a Left, scatterplot of H3K27me3 percentages along the body of expressed X-linked genes in female F3 ( $y$-axis) relative to male F2 ( $x$-axis) TS cells. Each dot represents a single gene and its respective percentage of H3K27me3 in male and female TS cells. $k$-means clustering was applied, which led to the identification of three classes of genes. Genes subject to I-XCI (red dots) are concentrated in the upper left quadrant consistently, with them being depleted in $\mathrm{H} 3 \mathrm{~K} 27 \mathrm{me} 3$ in male cells and enriched in $\mathrm{H} 3 \mathrm{~K} 27 \mathrm{me} 3$ in female cells as previously established [20]. Genes escaping from I-XCI in TS cells (blue dots) are significantly depleted in H3K27me3 in both male and female cells. In agreement with H3K27me3 marking preferentially the silent state, very few expressed genes are enriched in H3K27me3 in both male and female cells ([0] genes; indicated by maroon dots). These [0] genes may have resulted from the fact that gene expression data [29] and our ChIP-chip experiment data were obtained from different TS cell lines. Middle, scatterplot of H3K27me3 percentages along the body of expressed X-linked genes in the I-XCI revertant clone 1\#A ( $y$-axis) relative to female F3 ( $x$-axis) TS cells. Genes marked with red triangles "H3K27me3-low in 1\#A" represent genes that have not regain $\mathrm{H} 3 \mathrm{~K} 27 \mathrm{me} 3$ on the inactive $\mathrm{X}$ after the reversal of I-XCI patterns. Right, scatterplot of H3K27me3 percentages along the body of expressed X-linked genes in the I-XCl revertant clone 1\#A ( $y$-axis) relative to male F2 ( $x$-axis) TS cells. "H3K27me3-low in 1\#A" genes show a percentage of H3K27me3 that is not significantly different from the low levels of H3K27me3 on active allele in male TS cells by Kolmogorov-Smirnov test. The H3K27me3 profiles of Ndufa 1, Lamp2 and Atp7a analysed in panel $\mathbf{c}$ and $\mathbf{d}$ and of Cox7b and Hmgn5 analysed in Additional file 3C are shown. b Representative examples of H3K27me3 distribution along expressed genes in male, female and I-XCI clone 1\#A TS cells. University of California Santa Clara (UCSC) Genome Browser screenshots ( $m$ m8 build) are shown. IP immunoprecipitated DNA; IN input DNA. c Representative images of immuno-RNA-FISH for H3K27me3 (yellow) and Hprt7 (green) combined with the indicated X-linked gene (red) in F3 parental cells (upper panels) or in the indicated XCI revertant clones (lower pane/s). The chromosomal position of the genes analysed is depicted on the diagram on the left. In F3 cells, active alleles of Cybb, Ndufa 1, Lamp2 and Atp7a are located away from the H3K27me3 coated inactive X indicating that these genes are expressed from the active $X$. In revertant clones $1 \# B$ and $1 \# F$, active alleles of all four genes are located at the vicinity of the Hprt 1 signal marking the $X^{P}$ and away from the H3K27me3 accumulation which indicates that these genes are transcribed from the active X. Moreover, Cybb and Ndufa 1 are also transcribed, in these clones, from the inactive X as illustrated by a faint signal at the periphery of H3K27me3 accumulation (arrowheads). Maximal projection after deconvolution. Scale bar $5 \mu \mathrm{m}$. d Cumulative histograms of the percentages of nuclei with the depicted expression pattern in F3 TS cells and in nuclei of 4 independent $X \mathrm{Cl}$ revertant clones (analysis performed at passage 8 or later after clone picking). An Hprt1 signal (green pinpoint) identifies the $X^{P}$, while an $\mathrm{H} 3 \mathrm{~K} 27$ me3 domain marks the inactive $X$ (yellow domain). In XCI revertant clone $1 \# A$, X-linked genes Lamp2 and Atp7a that show high levels of H3K27me3 as assessed by ChIP-on-chip show a preferential expression from the reactivated $X^{P}$. In contrast, $X$-linked genes Cybb and Ndufar displaying low levels H3K27me3 by ChIP-on-chip show significant bi-allelic expression compared to F3 cells ( $X^{2}$ test $p$ value $<0.05$ ). No significant difference is observed amongst $X C l$ revertant clones ( $X^{2}$ test $p$ value $>0.05$ ). See Additional file 10 for probe position. $n>150$

accumulation of H3K27me3 on the inactive X (Fig. 3c, d). In nuclei of I-XCI revertant clones $1 \# \mathrm{~A}, 1 \# \mathrm{~B}, 2 \# \mathrm{~B}$ and 1\#F, Lamp 2 and Atp7a, two genes that show levels of H3K27me3 similar to F3 female cells (Fig. 3a), appeared to be transcribed from the active $\mathrm{X}^{\mathrm{P}}$ (i.e. lacking H3K27me3 and transcribing Hprt ${ }^{W T}$ ) in the majority of nuclei. In contrast, the Ndufa 1 locus, which shows low levels of H3K27me3 in 1\#A cells compared to F3 cells, exhibited significant bi-allelic expression in all revertant clones (20-30\% of nuclei). In addition, Ndufa1 active maternal alleles lacked H3K27me3 staining and appeared located at the periphery of H3K27me3 accumulation on the inactive $\mathrm{X}$. No Cybb signal could be detected in the majority of cells of XCI revertant clones (including 1 \#A cells) despite the fact that this gene displays low levels of H3K27me3 in these cells. Note that $C y b b$ is poorly expressed in F3 cells (as measured by RNA-FISH) and in TS cells in general (as assessed by gene expression microarray; see Additional file 4 and [33]). However, in the few cells expressing $C y b b$, the gene was transcribed from both X-chromosomes in a higher percentage of I-XCI revertant clone cells compared to the F3 parental cells (Fig. 3c, d).

We also tested whether low levels of H3K27me3 at some X-linked genes of 1 \#A cells were associated with significant expression from both $\mathrm{X}$-chromosomes using single-cell allelic RT-qPCR analysis. We measured the variability of expression levels of two H3K27me3-low genes, Cox $7 b$ and Hmgn5, amongst 1\#A clonal cells (see Additional file 3F). Cox $7 b$ appeared significantly expressed from the $\mathrm{X}^{\mathrm{M}}$-although at a lower level than from the $\mathrm{X}^{\mathrm{P}}$-in a subset of 1 \#A cells. In contrast, no such extensive leak of expression was observed from the maternal Hmgn5 allele on the same sample of cells.

These results indicate that the reversal of X-inactivation patterns is associated, at the vast majority of active $\mathrm{X}$-linked genes, with an enrichment of H3K27me3 on the inactive $\mathrm{X}^{\mathrm{M}}$. However, some specific genes resist H3K27me3 accumulation and are either expressed at low levels from the $\mathrm{X}^{\mathrm{M}}$ in a subset of cells or repress the maternal allele using a mechanism different from H3K27me3 recruitment.

\section{Female TS cells transiently pass through a state in which the two X-chromosomes are active before switching $\mathrm{X}$-inactivation profiles}

In order to understand how TS cells undergo this reversal of I-XCI, we analysed, by allelic RT-qPCR, the expression of Xist and of two other X-linked genes (Cox7b and $P g k 1$ ) at an earlier time point after clone isolation (in the presence of HAT selection). In the framework of the same HAT selection (experiment \#F), at passage 2 after clone picking, three categories of clones could be distinguished (Fig. 4). Two out of 40 clones already showed an inverted 

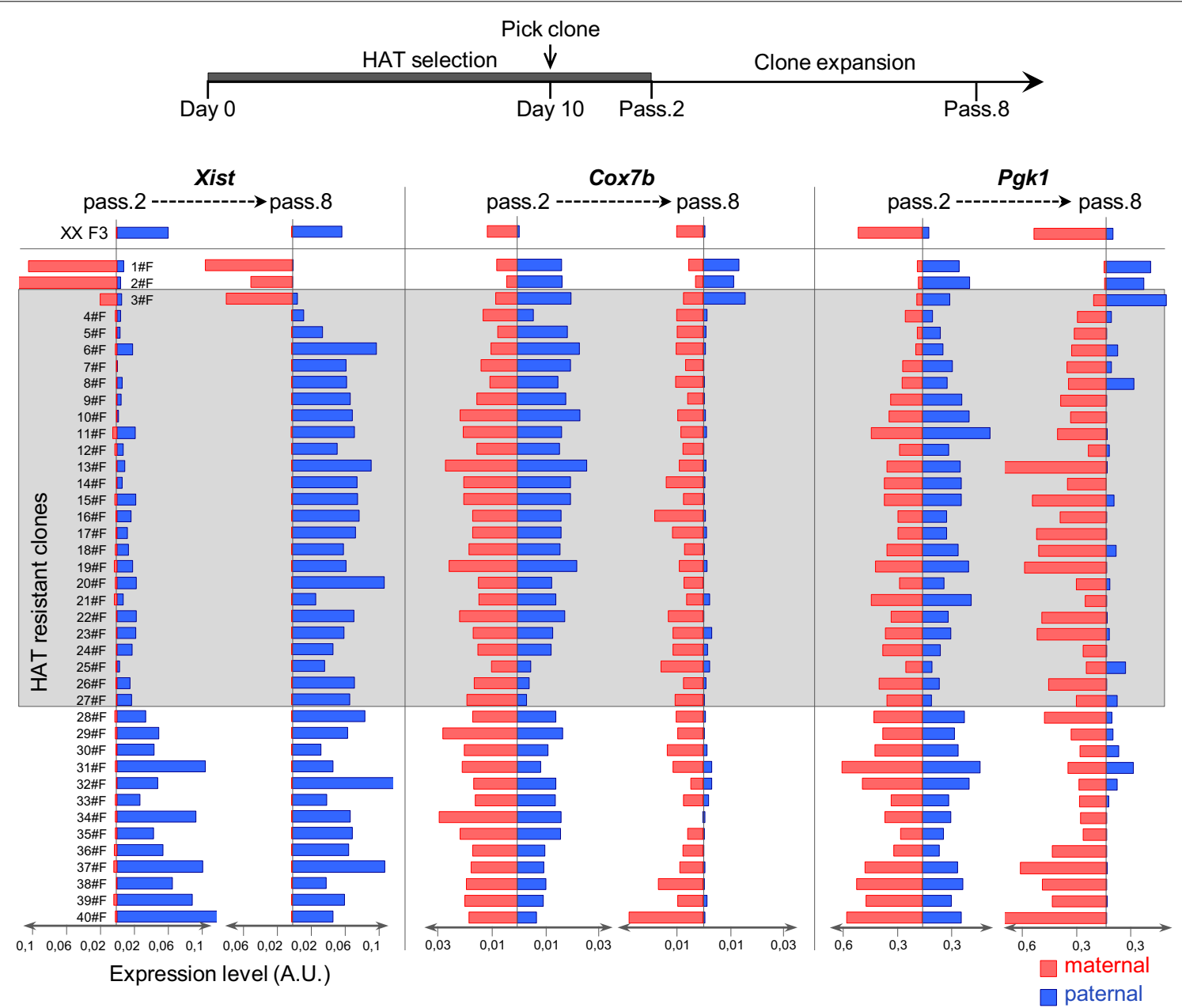

Fig. 4 Global reactivation of the paternal X precedes the switching of X-inactivation profiles in female TS cells. Histograms showing the paternal and maternal expression levels of Xist and X-linked genes Cox7b and Pgk1 in the indicated HAT-resistant clone assessed by allelic RT-qPCR. Two different time points during the selection procedure have been analysed: clones at passage 2 (pass.2) after clone picking (still under HAT selection) and the same clones after 6 additional passages without HAT (pass.8). Above the histograms, the diagram depicts the scheme of the HAT selection experiment used to isolate HAT-resistant clones analysed in this panel. Standardisation by Rplpo has been applied. A.U. Arbitrary Units

I-XCI profile, 25 clones displayed low levels of Xist expression overall combined with a bi-allelic expression of both Cox $7 b$ and Pgk1a, while 13 clones exhibited a preferential paternal expression of Xist associated with strong bi-allelic expression of Cox $7 b$ and $P g k 1$. Since this latter expression profile evolves, after HAT release (passage 8), towards a return to a parental-like XCI pattern, these clones have probably locally and transiently reactivated both $\mathrm{X}^{\mathrm{P}}$-linked loci together with the $\mathrm{Hprt1}^{W T}$ gene. As previously mentioned, similar transient, local and coordinated reactivations of several $\mathrm{X}$-linked loci on the inactive $\mathrm{X}^{\mathrm{P}}$ including Hprt1, Cox7b and Pgk1a have been observed in another TS cell line [20]. The fact that reactivation of specific X-linked genes takes place early during the selection process may indicate that the HAT treatment facilitates this phenomenon. We note, however, that these local relaxations of
X-linked silencing do not seem to be sufficient-at least on their own-to trigger the reversal of I-XCI since none of these clones evolved towards a global $\mathrm{X}^{\mathrm{P}}$ reactivation or $\mathrm{X}^{\mathrm{M}}$ inactivation upon HAT release.

In contrast, robust bi-allelic expression of X-linked genes accompanied by low-level Xist expression in the second category of clones may indicate the presence of two-active X-chromosomes. In order to test this hypothesis, we launched a new HAT selection and prolonged the HAT treatment with the hope of maintaining the cells under this putative two-active- $\mathrm{X}$ state (Additional file 5A; experiment $\# \mathrm{C}$ ). Under these conditions, we recovered the same categories of HAT-resistant clones with similar frequencies as previously observed (see Fig. 1b). We then concentrated on three clones $(1 \# \mathrm{C}, 2 \# \mathrm{C}$ and $3 \# \mathrm{C})$ that showed low levels of Xist expression under HAT pressure 
(passage 4 after clone picking) both in RT-qPCR and by Xist RNA-FISH (Additional file 5A, B). In these clones, we analysed the expression of $9 \mathrm{X}$-linked genes by allelic RT-qPCR at two different time points: passage 4 (under HAT) and passage 14 (i.e. 8 passages after HAT release). Coincidently with reduced Xist expression, a strong biallelic expression of most X-linked genes was observed in all three clones maintained in HAT. We noted, however, that Rps6ka6 retained a preferential expression from the $\mathrm{X}^{\mathrm{M}}$ in two clones, suggesting that the reactivation of paternal alleles of different X-linked genes may not occur completely synchronously in all clones. After HAT removal, 2 out of three clones evolved towards a maternal expression of X-linked genes associated with Xist paternal induction, while the third clone inactivated the maternal X-chromosome (Additional file 5A).

We conclude from these observations that the allelic switch of I-XCI patterns is probably mediated-at least in part-by a repression of paternal Xist expression associated with a global $\mathrm{X}^{\mathrm{P}}$ reactivation, which leads to the presence of two-active $\mathrm{X}$-chromosomes. We cannot, however, exclude that some TS cells directly switch from parental to inverted I-XCI upon HAT selection without transiting through a "two-active- $X$ " state. From the two-active- $X$ state, TS clones rapidly evolve, either towards a return to the parental-like configuration, or undergo I-XCI reversal.

\section{Allelic switch of I-XCI patterns does not require a transition through pluripotency}

Only two mouse cell types are known to tolerate a lack of $\mathrm{XCI}$ : the primordial germ cells and the cells of the inner cell mass of the blastocyst. This particularity may be related to the pluripotency of these cells (for review see [9, $34]$ and [15]). We thus tested whether the presence of twoactive X-chromosomes in HAT-resistant TS cells was associated with a conversion to a pluripotent state. To this end, we measured by RT-qPCR the expression level of pluripotency markers Oct3/4 and Nanog in TS clones maintained under HAT and shown to harbour two active Xs. No significant expression of either marker could be detected in such clones either at the population level (see Additional file 6A) or at the single-cell level (see Additional file 6B and Additional file 7). These clones still expressed trophectoderm-specific markers (see Additional file 6C, D) thereby indicating that TS cells do not detectably change cell-type identity upon reversal of I-XCI.

\section{The choice of which $X$ to inactivate after $X^{P}$ reactivation occurs synchronously and homogeneously within clonal TS cell populations showing two-active $\mathrm{X}$-chromosomes}

Theoretically, from the two-active- $\mathrm{X}$ state, individual TS cells may either choose to inactivate the $\mathrm{X}^{\mathrm{P}}$ or the $\mathrm{X}^{\mathrm{M}}$.
Under the hypothesis of a random, cell-autonomous, choice, we expect to observe mosaic clones consisting of cells of each configuration in number almost equal. Our results on TS clones after HAT release rather suggest a strong bias towards one or the other configuration depending on the clone. In order to determine precisely the mosaicism of clonal TS cell populations, we quantified the expression of Xist and of 5 other X-linked genes, at the single-cell level, using allelic RT-qPCR in 4 different clones: 2 clones showing a parental-like I-XCI (1\#C $\& 3 \# C$ ) and 2 clones showing an inverted I-XCI (1\#A \& 2\#C) after HAT removal (-HAT) (Fig. 5 and Additional files 7, 8 and 9). In agreement with our previous results on clonal cell populations, the vast majority of cells of clone $1 \# C$ and clone $3 \# C$ showed a homogeneous $\mathrm{XCI}$ profile with a preferential expression of maternal alleles of the genes we tested. The situation is less clear in clone $1 \# A$ and clone $2 \# C$ showing a switch of I-XCI in which we observed, depending on the gene, a subset of cells displaying a bi-allelic expression (i.e. Atp7a, Cox $7 b$ and Pgk1). Importantly, we did not observe, within a given clone, cells with the opposite XCI profile than the predominant configuration.

When we performed the same single-cell analysis on one clone of each category maintained under HAT pressure (3\#C and 2\#C (+HAT)), we observed low levels of Xist expression (see Additional file 8) associated with bi-allelic expression of most X-linked genes in most cells of clone $3 \# \mathrm{C}$ confirming the presence of two-active $\mathrm{X}$-chromosomes in those cells (Fig. 5, Additional file 9 and Additional file 7 for quantification results). In contrast, cells of clone $2 \# \mathrm{C}$ maintained under HAT pressure already showed a significant maternal Xist expression in $51 \%$ of the cells (see Additional file 8) accompanied by a heterogeneous behaviour amongst X-linked genes with some genes being bi-allelically expressed in most cells $(A t p 7 a, C o x 7 b)$, while others already showed a predominant paternal expression (Apool, Sh3bgrl) (Fig. 5 and Additional file 9). This suggests that the switch of $\mathrm{XCI}$ patterns is not synchronously established in all the cells of a given clone and at all X-linked genes of a given cell. Of note, we did not detect any marked cell death or higher levels of spontaneous differentiation during clone expansion that may be suggestive of a counter-selection or of a growth disadvantage of a subset of cells within clones.

In conclusion, the secondary biassed XCI we have observed here implies that the choice of which $\mathrm{X}$ to reinactivate in this cellular system of TS cells selected with HAT does not involve the same mechanism as the random choice of active and inactive X-chromosomes occurring upon ES cell and ICM differentiation. 

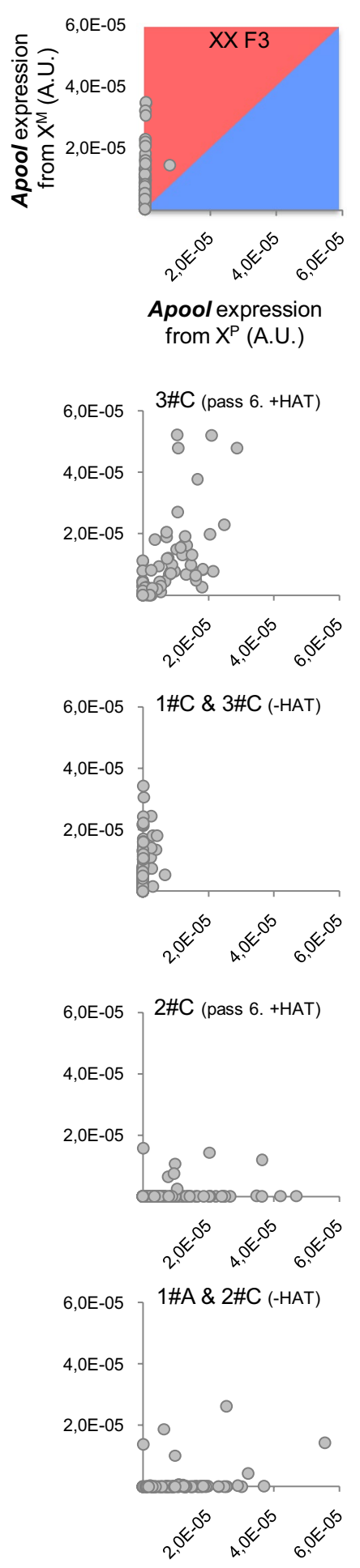
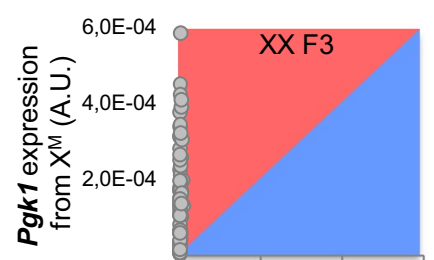

\section{Parental cell line}

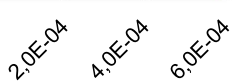

Pgk1 expression from $X^{P}$ (A.U.)
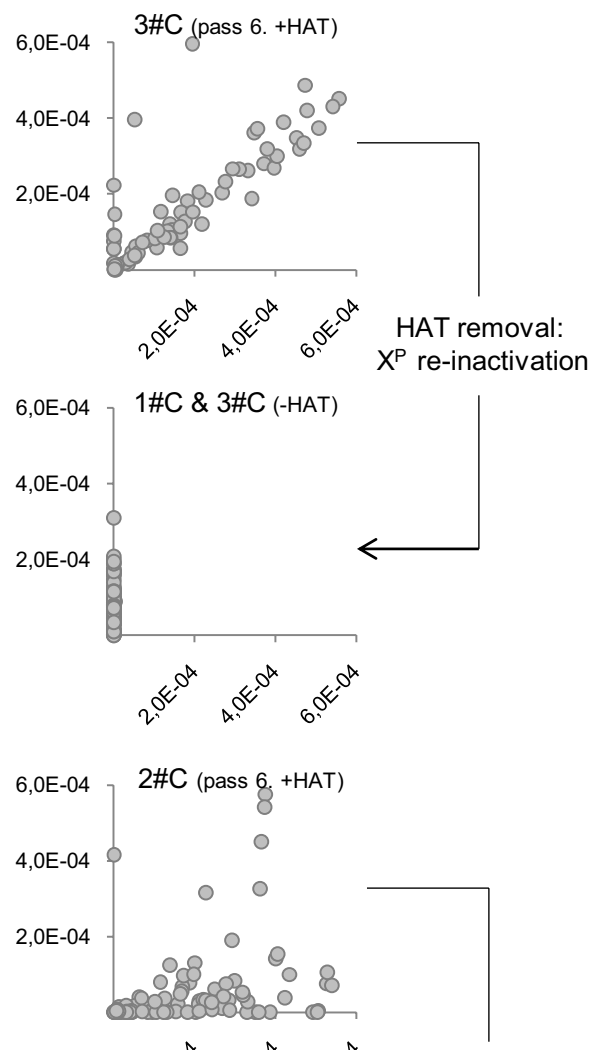

HAT removal:

Reversal of $\mathrm{I}-\mathrm{XCl}$

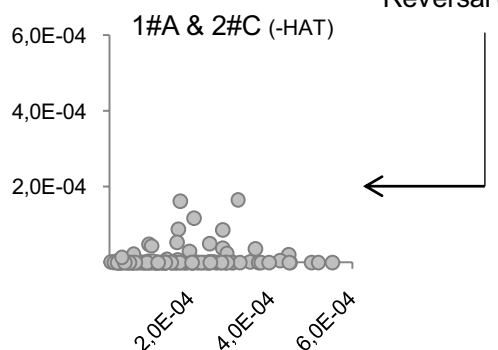

Fig. 5 Biased X-inactivation occurs upon HAT release in clonal cell populations harbouring two-active X-chromosomes. Scatterplots of expression levels from the paternal ( $x$-axis) relative to the maternal ( $y$-axis) X-chromosome for the indicated gene in individual cells of the indicated clones assessed by allelic RT-qPCR (BioMark, Fluidigm). Each dot represents a single TS cell. (+HAT): cells at passage 6 maintained under HAT pressure; (HAT): cells after HAT removal. The same samples of cells have been analysed for the expression of the genes shown in this figure and in Additional files 8 and 9. F3: $n=77 ; 3 \# C$ (+HAT): $n=75 ; 2 \# C(+H A T): n=108 ; 1 \# C \& 3 \# C(-H A T): n=144 ; 1$ \#A \& 2\#C $(-$ HAT): $n=141$. (See Additional file 7) for raw quantifications. A.U. Arbitrary Units 


\section{Discussion}

Here, we show that trophoblast stem cells derived from the polar trophectoderm exhibit a remarkable plasticity allowing them to reactivate the paternal $\mathrm{X}$ and to almostfully invert I-XCI profiles (see Additional file 11A). Simi$\operatorname{lar} \mathrm{X}^{\mathrm{P}}$ reactivation or reversal of I-XCI had already been observed in vivo in mutant contexts in which Xist regulation had been impaired [26, 35]. Our results further suggest that trophoblast cells can undergo this allelic switch in the absence of experimentally induced Xist deregulation. This plasticity of I-XCI in the trophectoderm may serve as a back-up mechanism allowing placental progenitors to promptly adapt to mutations affecting genes on the maternal $\mathrm{X}$ or to environmental pressure favouring the usage of one allelic form compared to the other, like in the present study, in which only the cells expressing the paternal Hprt1 allele can survive in a HAT-containing medium. In the case of the present study, it is also possible that the HAT treatment represents a form of stress for the cells that may modify some layers of epigenetic regulation thereby facilitating the changes of XCI profiles. More generally, the plasticity of I-XCI may also be at work in the case of dysfunction of $\mathrm{X}^{\mathrm{P}}$ silencing in order to maintain a single dose of $\mathrm{X}$-linked gene products. It may also be responsible for inactivating one of the two maternal Xs in $\mathrm{X}^{\mathrm{M}} \mathrm{X}^{\mathrm{M}}$ parthenogenetic embryos $[1,36,37]$ (see Additional file 11B).

To undergo the switch of I-XCI profiles, some-if not all-TS cells globally reactivate their paternal $X$ to reach a two-active-X state. This transition correlates with a reversal of epigenetic mechanisms maintaining the inactive state including a loss of Xist coating and a resetting of histone modifications specific to the inactive $\mathrm{X}$ such as H3K27me3. The two-active-X state appears extremely labile indicating that TS cells cannot stand a double dose of X-linked gene expression for multiple cell divisions. Interestingly, the EED component of the PRC2 complex-the complex responsible for adding and maintaining H3K27me3 on the inactive $\mathrm{X}$-becomes necessary to maintain $\mathrm{X}^{\mathrm{P}}$ silencing only after TS cell differentiation $[38,39]$. This suggests that the silencing of the paternal $\mathrm{X}$ is not fully stabilised in undifferentiated TS cells which may facilitate the reactivation of this chromosome and results in the presence, at a low frequency, of TS cells exhibiting active paternal alleles among the global TS cell population. Alternatively and not exclusively, the $K d m 6 a / U t x \mathrm{X}$-linked gene, which escapes from X-inactivation and encodes an H3K27me3 demethylase, may be responsible for the H3K27 demethylation of the paternal $\mathrm{X}$ which accompanies the reversal of I-XCI profiles in TS cells. Indeed, Kdm6a/Utx has been shown to be necessary for somatic cell reprogramming towards pluripotency [40], which is accompanied by a reactivation of the inactive $\mathrm{X}$, and H3K27me3 demethylase activities participate in the repression of Xist in ES cells [41].

Collectively, these features evoke the dynamic turnover-erasure and de novo establishment-of methylation marks used in human ES cells to maintain DNA methylation profiles, which leads to ES cell populations showing heterogeneous methylation patterns. In contrast, in differentiated cells, methylation marks are stably maintained through copying them from mother to daughter cells, a mechanism that is thought to be more vulnerable to the insertion and to the propagation of aberrant methylation marks in the cell population [42]. Following this reasoning, one may speculate that the ease of reactivation of $\mathrm{X}^{\mathrm{P}}$ alleles in undifferentiated TS cells may be permitted by similar ongoing turnover of epigenetic marks such as H3K27me3.

From the two-active-X metastable state, the cells evolve either towards a return to the parental profile or towards a switch of I-XCI profiles. Unexpectedly, our analysis shows that within clonal TS cell populations displaying two active Xs, sister cells homogeneously choose the same inactivation path as illustrated by the lack of mosaic clones comprising cells that have inactivated the $\mathrm{X}^{\mathrm{P}}$ mixed with cells that, conversely, have silenced the $\mathrm{X}^{\mathrm{M}}$ (see Additional file 11B). The choice of which $\mathrm{X}$ to inactivate after $\mathrm{X}^{\mathrm{P}}$ reactivation in TS cells therefore differs from the random choice mechanism recruited during ES cell differentiation. In addition, the process of $\mathrm{X}^{\mathrm{P}}$ reactivation followed by $\mathrm{X}^{\mathrm{P}}$ re-inactivation occurs synchronously at most $\mathrm{X}$-linked genes and between sister cells, while the de novo inactivation of the $\mathrm{X}^{\mathrm{M}}$ appears more chaotic and is more rapidly established. Furthermore, while $\mathrm{X}^{\mathrm{P}}$ re-inactivation is only observed after HAT removal, $\mathrm{X}^{\mathrm{M}}$ silencing may take place under HAT pressure. These results suggest that clones returning to the parental profile have retained a memory of the inactive state that inhibits I-XCI reversal and may explain why I-XCI reversal occurs less frequently. This is reminiscent of the memory of the inactive state observed in cells of the trophoblast lineage of cloned embryos originating from somatic cell nuclear transfer [43]. In contrast, the stochastic behaviour of TS cells showing a switch of I-XCI supports the hypothesis that the maternal imprint that presumably prevents $\mathrm{X}^{\mathrm{M}}$ inactivation has been erased-at least partially-in these clones and that the HAT pressure favours the inactivation of the $\mathrm{X}^{\mathrm{P}}$. In these cells, some genes on the $\mathrm{X}^{\mathrm{M}}$ however escape both from de novo H3K27trimethylation and from X-wide silencing indicating that they may be refractory to inactivation or that a tight control of their expression is not crucial for TS cell homeostasis. Alternatively, these non-cell-autonomous behaviours suggest cell-to-cell communications within clonal cell populations. 


\section{Conclusions}

In summary, TS cells appear to be able to reprogram $\mathrm{X}$-chromosome expression back and forth without any detectable change in cell-type identity. Notably, the reactivation of the paternal $\mathrm{X}$ is not associated with a conversion to a pluripotent state as opposed to reactivation of the inactive $\mathrm{X}$ in the embryonic lineage, in the germline, or during somatic cell reprogramming into iPS cells. This suggests that these stem cells show unique epigenomic features facilitating the reprogramming of gene expressions and thereby enabling trophoblast stem cells to adapt to any impairment of programmed mono-allelic gene expression that may occur during extraembryonic development. Such specific features put forward the TS cell model as an alternative background to explore lineagespecific determinants of genome plasticity/multipotency.

\section{Methods}

\section{TS cell culture and HAT/6-TG treatments}

Male F2 and female F3 TS cell lines used in this study were derived in the lab and have been previously described $[12,13]$. F3 cells carry an $\mathrm{X}^{\mathrm{P}}$ from $129 / \mathrm{Sv}$ origin and an $\mathrm{X}^{\mathrm{M}}$ from 129.Pgk1a origin providing polymorphisms allowing for allelic distinction. TS culture conditions were as previously described [12, 13, 44]. Cells were pre-treated or not with $30 \mu \mathrm{M}$ of 6-TG (Sigma A4882) for a week then treated with HAT $(100 \mu \mathrm{M}$ Hypoxanthine, $0.4 \mathrm{mM}$ Aminopterin, $16 \mathrm{mM}$ Thymidine) for 10 days. Cells were then grown for two additional passages in medium containing HT but lacking Aminopterin, which is the component assimilated by HPRT, to allow TS cells to recover from the selection before they are grown in standard TS medium again. The frequency of HAT-resistant cells within the framework of a given HAT selection corresponds to the number of HAT-resistant clones observed at the end of the selection divided by the number of TS cells initially plated. The average frequency of HAT resistance corresponds to the mean of frequencies observed in the time course of independent selection experiments (\#A, \#B, \#C, \#D, \#E and \#F).

\section{ChIP-chip procedure and data analysis}

ChIP assays were performed as previously described [45] using an H3K27me3 antibody (07-449 from EMD Millipore, Billerica, MA, USA) and analysed as described previously $[19,20]$. ChIP-chip data have been deposited under Gene Expression Omnibus accession number [GSE:68536]. Two replicates of each experiment are available and show similar results.

Raw data were analysed using the Tobias Straub protocol (http://www.epigenesys.eu/images/stories/protocols/ pdf/20111025114444_p43.pdf) with the Bioconductor R interface. This protocol includes quality assessment, data normalisation and transformation. The gene expression data of TS cells were extracted from [GSE:15519] [33].

\section{RNA-FISH and Immuno-RNA-FISH}

RNA-FISH and immuno-RNA-FISH procedures were carried out as described in [46] (H3K27me3 antibody, 07-449; EMD Millipore). For details of our experimental procedures, for probe localisation and for image capture protocol, see [19].

\section{Single-cell gene expression analysis}

Single-cell gene expression analysis was performed as described previously in $[19,20]$ and as recommended by Fluidigm (http://www.fluidigm.com/single-cell-expression.html; South San Francisco, CA, USA). Briefly, TS cells were sorted by fluorescence-activated cell sorting using the MoFlo system (Beckman Coulter, Brea, CA, USA), and individual cells were distributed into wells or 96-well plates containing $5 \mu$ of CellsDirect resuspension buffer (Invitrogen, Carlsbad, CA, USA). The preamplification step consisted of 20 cycles using a mix of universal primer pairs to preamplify each gene simultaneously. Preamplification was followed by exonuclease I treatment (New England Biolabs, Ipswich, MA, USA), and allelic qPCR was performed on a BioMark thermal cycler (Fluidigm). Raw efficiencies of each PCR assay and allelic specificity were measured on control DNA within each experiment. Transcript levels were extrapolated using the raw PCR efficiencies, thus allowing the direct comparison of different genes. Controls for allelic specificities of PCR assays are available upon request. Three housekeeping control RNAs (Gapdh, Hist $2 h 2 a$ and Rplpo) were systematically quantified in parallel in each single cell. See Additional file 7 for raw quantifications. Detailed analyses and statistical tests have been performed using the Qlucore Omics Explorer 2.3 (QLUCORE Company). For primer sequences, see [19].

\section{Additional files}

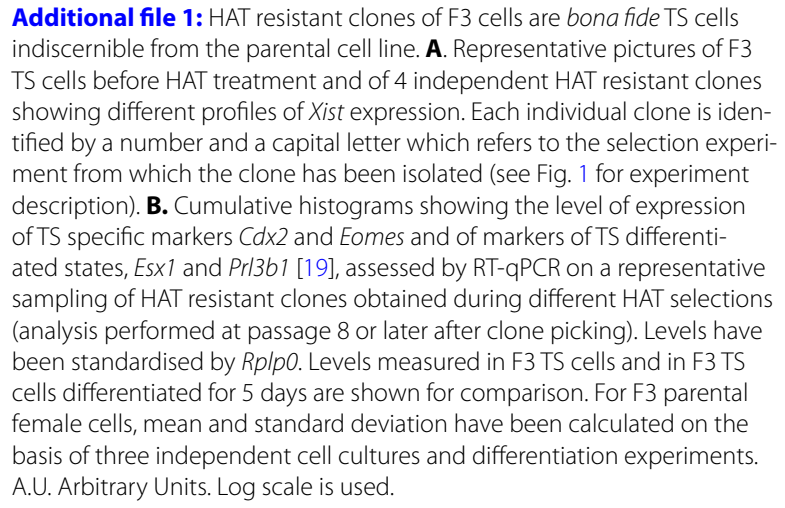

Additional file 1: HAT resistant clones of F3 cells are bona fide TS cells indiscernible from the parental cell line. A. Representative pictures of F3 TS cells before HAT treatment and of 4 independent HAT resistant clones showing different profiles of Xist expression. Each individual clone is identified by a number and a capital letter which refers to the selection experiment from which the clone has been isolated (see Fig. 1 for experiment description). B. Cumulative histograms showing the level of expression of TS specific markers $C d \times 2$ and Eomes and of markers of TS differentiated states, Esx 1 and Pr/3b1 [19], assessed by RT-qPCR on a representative sampling of HAT resistant clones obtained during different HAT selections (analysis performed at passage 8 or later after clone picking). Levels have been standardised by Rplp0. Levels measured in F3 TS cells and in F3 TS cells differentiated for 5 days are shown for comparison. For F3 parental female cells, mean and standard deviation have been calculated on the basis of three independent cell cultures and differentiation experiments. A.U. Arbitrary Units. Log scale is used. 
Additional file 2: X-linked gene expression profiles in various types of HAT resistant clones. A. RNA-FISH for Xist (red) in some of the clones depicted in Fig. 1C. Representative images and cumulative histrograms of the percentage of nuclei with the depicted expression pattern are shown (analysis performed at passage 8 or later after clone picking). No significant difference is observed between any of the clones and F3 parental cells ( $X^{2}$ test). $n>100$. Scale bar $=5 \mu \mathrm{m}$. B. Allelic RT-qPCR analysis of $X$-linked gene expression in HAT resistant clones showing an F3-like expression profile (analysis performed at passage 8 or later after clone picking). Cumulative histograms showing the paternal (blue) and maternal (red) expression levels of the indicated X-linked are shown. Standardisation by Rplpo has been applied. For F3 parental female cells, mean and standard deviation have been calculated on the basis of three independent cell cultures. The chromosome diagram on the left shows the localisation of the genes analysed in RT-qPCR and by RNA-FISH in panel B, C and D. C. Cumulative histograms of the percentages of nuclei with the depicted RNA-FISH expression pattern in F3 TS cells and in nuclei of 3 independent HAT resistant clones showing a parental-like Xist expression profile (analysis performed at passage 8 or later after clone picking). Seven different $\mathrm{X}$-linked genes distributed along the $\mathrm{X}$-chromosome have been analysed (see Additional file 10 for probe coordinates). Representative images of double RNA-FISH for Xist and for the indicated X-linked gene in clones $12 \# F$ and $23 \# F$ are shown on the right hand side of the histogram. The inactive $X$ chromosome is identified through Xist RNA accumulation (red). Primary transcription at the indicated X-linked gene is co-detected in green. $<1 \%$ of $\mathrm{F} 3$ parental TS cells show an Hprt 1 signal in agreement with the inactivation of the wild type, paternal, Hprt1 allele. In contrast, an Hprt1 signal associated with the Xist domain is detected in 25 to $50 \%$ of nuclei of HAT resistant clones $12 \# \mathrm{~F}, 23 \# \mathrm{~F}$ and $36 \# \mathrm{~F}$ indicating that these clones have locally reactivated the Hprt $1^{W T}$ allele on the inactive paternal $X$. Note that active Hprt1 alleles appear to extrude from the Xist domain in these category of HAT resistant clones. No significant difference in the expression profiles is observed between any of the clones and F3 parental cells at X-linked genes Tfe3, Lamp2, Atp7a, Pgk1, Fgd1 and the X-inactivation escaper $K d m 5 c\left(X^{2}\right.$ test, $p$ value $\left.>0.05\right) . n>150$. Scale bar $=5 \mu \mathrm{m}$. D. Allelic RT-qPCR analysis of Cox7b and Atp7a in 8 clones showing an allelic inversion of Xist expression profiles (analysis performed at passage 8 or later after clone picking). Cumulative histograms showing the paternal (blue) and maternal (red) expression levels of the indicated X-linked are shown. Standardisation by Rplpo has been applied. For F3 parental female cells, mean and standard deviation have been calculated on the basis of three independent cell cultures.

Additional file 3: $\mathrm{H} 3 \mathrm{~K} 27 \mathrm{me} 3$ accumulates on the inactive $\mathrm{X}$ chromosome in $\mathrm{I}-\mathrm{XCl}$ revertant clones. A. Representative image of immunostaining followed by RNA-FISH for H3K27me3 (green) and Xist (red) on female F3 TS cells and on cells of $2 \mathrm{I}-\mathrm{XCl}$ revertant clones. Scale bar $=5 \mu \mathrm{m}$. $\mathbf{B}$. Cumulative histogram showing the percentage of nuclei exhibiting an accumulation of Xist RNA only (red), co-accumulation of Xist RNA and H3K27me3 (yellow) and accumulation of H3K27me3 only (green) in F3 TS cells and in cells of I-XCI revertant clones 1\#A and 1\#C (analysis performed at passage 8 or later after clone picking). No significant difference is observed between any of the clones and $\mathrm{F} 3$ parental cells ( $\mathrm{X}^{2}$ test). $n>100$. C. Boxplots of the distribution of H3K27me3 along expressed $\mathrm{X}$-linked genes in male F2 TS cell, in F3 female TS cells and in cells of I-XCI revertant clone 1\#A (analysis performed at passage 8 or later after clone picking)(see Additional file 4 for gene by gene percentage of enrichment). Expression data were extracted from the Gene Expression Omnibus database [GSE:15519] [33]. $n=203$ expressed X-linked genes. ${ }^{*} p$-value $<0.05$ by Kolmogorov-Smirnov test. D. Boxplots of the distribution of H3K27me3 along X-linked intergenic probes. $n=263245$ intergenic probes. No significant difference between the 3 cell lines was observed (Kolmogorov-Smirnov test). E. Boxplots of the distribution of H3K27me3 along the body of $\mathrm{X}$-linked genes that are not significantly expressed in TS cells. Expression data were extracted from the Gene Expression Omnibus database [GSE:15519] [33]. $n=292$ X-linked genes. No significant difference between the 3 cell lines was observed (Kolmogorov-Smirnov test). F. Scatterplot of expression levels from the paternal ( $x$-axis) relative to the maternal ( $y$-axis) $X$ chromosome for the indicated gene in 56 individual cells of the 1 \#A I-XCI revertant clone assessed by allelic RT-qPCR (analysis performed at passage 8 or later after clone picking). Each dot represents a single TS cell. Similar results have been obtained on another $\mathrm{I}-\mathrm{XCl}$ revertant clone. Expression levels have been normalised by the median value for each gene. A.U.: Arbitrary Units.

Additional file 4: ChIP-on-chip analysis of H3K27me3 distribution along $X$-linked genes in male F2 TS cells, in female F3 TS cells and in I-XCI revertant cells from clone 1\#A. Mean of two replicate ChIP experiments.

Additional file 5: Global reactivation of the paternal X precedes the switching $X$-inactivation profiles in female TS cells. A. Cumulative histograms showing the expression levels of paternal (blue) and maternal (red) alleles of the indicated gene, assessed by allelic RT-qPCR, in parental F3 cells and in 3 independent clones showing low levels of Xist RNA. Two time points have been analysed: clones at passage 4 after clone picking and still under HAT pressure (Pass.4 histograms) and the same clones, 8 passages after HAT release (Pass.14 histograms). Note that, in contrast with other $\mathrm{X}$-linked genes, Pbdc1 maintains a bi-allelic expression after HAT release in agreement with its status of $\mathrm{XCl}$ escaper. Standardisation by Rplpo followed by standardisation by the median value for each gene have been applied to allow direct comparison of the different genes on the same histogram. For F3 parental female cells, mean and standard deviation have been calculated on the basis of three independent cell cultures. Above the histograms, the diagram depicts the scheme of the HAT selection experiment used to isolate HAT resistant clones analysed in this panel. A.U. : Arbitrary Units. B. Representative image of Xist RNA-FISH on nuclei of clone $2 \# C$ and clone $3 \# C$ maintained under HAT pressure. On the right, the histogram shows the percentages of nuclei of each depicted category. Clones under HAT pressure harbour significantly different Xist expression profiles compared to F3 parental cells ( $X^{2}$ test $p$-value $<0.05$ ). $n>150$.

Additional file 6: The two-active-X state of early HAT resistant clones is not associated with cell reprogramming towards pluripotency. $\mathbf{A}$. Cumulative histograms showing the expression levels of pluripotency markers Nanog and Oct3/4 measured by RT-qPCR in F3 parental female TS cells and in 3 clones harbouring two active $X$-chromosomes. Expression levels in XX ES cells (LF2 cell line) are shown for comparison. Standardisation by Rplpo has been applied. A.U.: Arbitrary Units. B. Same as in panel A on individual cells of each population. Small horizontal bars represent median values. See Additional file 7 for raw quantifications. C. Cumulative histograms showing the level of expression of TS specific markers $C d \times 2$ and Eomes and of markers of TS differentiated states, Esx1 and Pr/3b1 [19], assessed by RT-qPCR on a the same clones as in panel A. Expression levels have been standardised by Rplp0. Expression levels measured in F3 TS cells and in F3 TS cells differentiated for 5 days are shown for comparison. A.U. Arbitrary Units. Log scale is used. D. Same as in panel $\mathbf{C}$ for $C d x 2$ and Eomes on individual cells of each population. Small horizontal bars represent median values. See Additional file 7 for raw quantifications.

Additional file 7: Single-cell RT-qPCR analysis in F3 parental cells, in cells of clones 1 \#C and 1\#A after HAT removal and in cells of clones $3 \# C$ and 2\#C both before and after HAT release.

Additional file 8: Single-cell analysis of Xist expression in clonal cell populations under HAT selection and after HAT removal. Scatter-plots of Xist expression levels from the paternal ( $x$-axis) relative to the maternal ( $y$-axis) X chromosome in individual cells of the indicated clones assessed by allelic RT-qPCR (BioMark, Fluidigm). Each dot represents a single TS cell. (+HAT): cells at passage 6 maintained under HAT pressure; (-HAT): cells after HAT removal. The same samples of cells have been analysed for the expression of the genes shown in Fig. 5 and in Additional file 9. For clones showing parental-like profiles or inverted I-XCl after HAT removal, cells from two different clones are shown. No significant difference was detected between cells of these two clones (Kolmogorov-Smirnov test). In clone $3 \# C$ under HAT pressure, one cells out of 74 tested show a significant Xist expression ( $>0.510^{-5}$ A.U. corresponding to level of Xist RNA molecules in a Xist domain), while, in clone $2 \# C$ under HAT pressure, 55 cells out of 108 tested show a significant Xist expression. F3: $\mathrm{n}=77$; $3 \# C(+H A T): n=75 ; 2 \# C(+H A T): n=108 ; 1 \# C \& 3 \# C(-H A T): n=144 ; 1 \# A$ $\& 2 \# C(-H A T): n=141$. An additional table shows the raw quantification results (see Additional file 7). A.U.: Arbitrary Units. 
Additional file 9: Single-cell analysis of gene expression of X-linked gene Atp7a, Cox7b and Sh3bgrl in clonal cell populations under HAT selection and after HAT removal. For details (see Additional file 8). See Additional file7 for raw quantifications.

Additional file 10: Name and chromosomal position of X-linked RNAFISH probes used in the study.

Additional file 11: Summary of the metastable states of I-XCl in female TS cells observed in the present study. A. Table summarising the characteristics of the various TS cell populations observed in the study. B. We observe in the present study that undifferentiated TS cells spontaneously reactivate the paternal $\mathrm{X}$ partially or completely at a low frequency. Under specific environmental cues (including, in the case of the present study, the HAT selection) or mutation that either prevent $X^{P}$ inactivation or favour the expression of $X^{P}$ genes, TS cells carrying two active $X$ chromosomes may undergo de novo inactivation of the maternal $X$.

\section{Abbreviations}

$X^{\mathrm{M}}$ : maternal $X$ chromosome; $X^{P}$ : paternal $X$ chromosome; TS cells: trophoblast stem cells; ES cells: embryonic stem cells; XEN cells: extraembryonic endoderm stem cells; ICM: inner cell mass; I-XCl: imprinted X-chromosome inactivation; R-XCl: random X-chromosome inactivation; HAT: Hypoxanthine, Aminopterin, Thymidine; RNA-FISH: fluorescence in situ hybridisation on ribonucleic acid; RT-qPCR: reverse transcription followed by quantitative polymerase chain reaction; ChIP: chromatin immuno precipitation.

\section{Authors' contributions}

JP performed most the HAT treatments, performed the molecular analyses including RT-qPCR on cell population, single-cell RT-qPCR analyses and FISH analyses, analysed the results and corrected the manuscript; AD participated in some HAT treatments, performed the TS cell cultures for the ChIP experiments, analysed the ChIP-on-chip data and corrected the manuscript; PN performed the ChIP-on-chip experiments and corrected the manuscript; DA isolated the TS cell lines and performed the first HAT treatment; PA designed the experiments, analysed the results and corrected the manuscript; CM designed the experiments, analysed the results, supervised the project, wrote the manuscript and performed experiments for the manuscript revision. All authors read and approved the final manuscript.

\section{Author details}

${ }^{1}$ Mouse Molecular Genetics Laboratory, Pasteur Institute, 25 rue du Dr Roux, 75015 Paris, France. ${ }^{2}$ Epigenetics of Stem Cells Laboratory, Pasteur Institute, 25 rue du Dr Roux, 75015 Paris, France. ${ }^{3}$ Dynamics of Epigenetic Regulation, EMBL Monterotondo, Adriano Buzzati-Traverso Campus, Via Ramarini 32, 00015 Monterotondo, Italy. ${ }^{4}$ CNRS, UMR7216 Epigenetics and Cell Fate, 35 rue Hélène Brion, 75013 Paris, France.

\section{Acknowledgements}

We thank the Centre d'Immunologie Humaine ( $\mathrm{ClH}$-Human Immunology Centre) for allowing and facilitating the use of the Biomark thermocycler. We thank Ann Holmberg from the QLUCORE Company for her help with the analysis of single-cell RT-qPCR results. We thank Graham Hayhurst for critical reading of the manuscript. This work was supported by recurrent funding from the Pasteur Institute and from the French National Centre for Scientific Research (CNRS) and by grants from the French National Agency for Research (ANR), the Epigenome Network of Excellence, the REVIVE Labex and the Louis D Foundation of the Institut de France (Louis D Prize awarded to PA). J.P. was supported by a doctoral fellowship from the Région Ile-de-France (DIM-Biothérapies), and by a grant from the REVIVE Labex. PN and AD are supported on a permanent basis by the Pasteur Institute. CM is supported on a permanent basis by the French National Institute for Scientific and Medical Research (INSERM).

\section{Competing interests}

The authors declare that they have no competing interests.

Received: 24 July 2015 Accepted: 16 November 2015

Published online: 01 December 2015

\section{References}

1. Miri K, Latham K, Panning B, Zhong Z, Andersen A, et al. The imprinted polycomb group gene Sfmbt2 is required for trophoblast maintenance and placenta development. Development. 2013;140:4480-9.

2. Okamoto I, Arnaud D, Le Baccon P, Otte AP, Disteche CM, et al. Evidence for de novo imprinted X-chromosome inactivation independent of meiotic inactivation in mice. Nature. 2005;438:369-73.

3. Okamoto I, Otte AP, Allis CD, Reinberg D, Heard E. Epigenetic dynamics of imprinted $X$ inactivation during early mouse development. Science. 2004;303:644-9.

4. Patrat C, Okamoto I, Diabangouaya P, Vialon V, Le Baccon P, et al. Dynamic changes in paternal X-chromosome activity during imprinted X-chromosome inactivation in mice. Proc Natl Acad Sci USA. 2009;106:5198-203.

5. Mak W, Nesterova TB, de Napoles M, Appanah R, Yamanaka S, et al. Reactivation of the paternal $X$ chromosome in early mouse embryos. Science. 2004;303:666-9.

6. Takagi N, Sasaki M. Preferential inactivation of the paternally derived X chromosome in the extraembryonic membranes of the mouse. Nature. 1975;256:640-2.

7. West JD, Frels WI, Chapman VM, Papaioannou VE. Preferential expression of the maternally derived $X$ chromosome in the mouse yolk sac. Cell. 1977; 12:873-82.

8. Monk M, Harper MI. Sequential X chromosome inactivation coupled with cellular differentiation in early mouse embryos. Nature. 1979;281:311-3.

9. Augui S, Nora EP, Heard E. Regulation of X-chromosome inactivation by the X-inactivation centre. Nat Rev Genet. 2011;12:429-42.

10. Wutz A. Gene silencing in X-chromosome inactivation: advances in understanding facultative heterochromatin formation. Nat Rev Genet. 2011;12:542-53.

11. Evans MJ, Kaufman MH. Establishment in culture of pluripotential cells from mouse embryos. Nature. 1981;292:154-6.

12. Tanaka S, Kunath T, Hadjantonakis AK, Nagy A, Rossant J. Promotion of trophoblast stem cell proliferation by FGF4. Science. 1998;282:2072-5.

13. Kunath T, Arnaud D, Uy GD, Okamoto I, Chureau C, et al. Imprinted X-inactivation in extra-embryonic endoderm cell lines from mouse blastocysts. Development. 2005;132:1649-61.

14. Navarro P, Chambers I, Karwacki-Neisius V, Chureau C, Morey C, et al. Molecular coupling of Xist regulation and pluripotency. Science. 2008;321:1693-5.

15. Schulz EG, Meisig J, Nakamura T, Okamoto I, Sieber A, et al. The two active $X$ chromosomes in female ESCs block exit from the pluripotent state by modulating the ESC signaling network. Cell Stem Cell. 2014;14:203-16.

16. Hoki Y, Ikeda R, Mise N, Sakata Y, Ohhata T, et al. Incomplete X-inactivation initiated by a hypomorphic Xist allele in the mouse. Development. 2011;138:2649-59.

17. Marahrens Y, Panning B, Dausman J, Strauss W, Jaenisch R. Xist-deficient mice are defective in dosage compensation but not spermatogenesis. Genes Dev. 1997;11:156-66.

18. Calabrese JM, Sun W, Song L, Mugford JW, Williams L, et al. Site-specific silencing of regulatory elements as a mechanism of $X$ inactivation. Cell. 2012;151:951-63.

19. Merzouk S, Deuve JL, Dubois A, Navarro P, Avner P, et al. Lineage-specific regulation of imprinted $X$ inactivation in extraembryonic endoderm stem cells. Epigenetics Chromatin. 2014;7:11.

20. Dubois A, Deuve JL, Navarro P, Merzouk S, Pichard S, et al. Spontaneous reactivation of clusters of $X$-linked genes is associated with the plasticity of $X$-inactivation in mouse trophoblast stem cells. Stem Cells. 2014;32:377-90.

21. Corbel C, Diabangouaya P, Gendrel AV, Chow JC, Heard E. Unusual chromatin status and organization of the inactive $X$ chromosome in murine trophoblast giant cells. Development. 2013;140:861-72.

22. Hadjantonakis AK, Cox LL, Tam PP, Nagy A. An X-linked GFP transgene reveals unexpected paternal $X$-chromosome activity in trophoblastic giant cells of the mouse placenta. Genesis. 2001;29:133-40.

23. Hemberger M, Kurz H, Orth A, Otto S, Luttges A, et al. Genetic and developmental analysis of $X$-inactivation in interspecific hybrid mice suggests a role for the $Y$ chromosome in placental dysplasia. Genetics. 2001;157:341-8.

24. Tam PP, Williams EA, Tan SS. Expression of an X-linked HMGlacZ transgene in mouse embryos: implication of chromosomal 
imprinting and lineage-specific X-chromosome activity. Dev Genet. 1994;15:491-503.

25. Tan SS, Williams EA, Tam PP. X-chromosome inactivation occurs at different times in different tissues of the post-implantation mouse embryo. Nat Genet. 1993;3:170-4.

26. Mugford JW, Yee D, Magnuson T. Failure of extra-embryonic progenitor maintenance in the absence of dosage compensation. Development. 2012;139:2130-8.

27. Hemberger $\mathrm{M}$. The role of the $\mathrm{X}$ chromosome in mammalian extra embryonic development. Cytogenet Genome Res. 2002;99:210-7.

28. Kuehn MR, Bradley A, Robertson EJ, Evans MJ. A potential animal model for Lesch-Nyhan syndrome through introduction of HPRT mutations into mice. Nature. 1987;326:295-8.

29. Berletch JB, Yang F, Xu J, Carrel L, Disteche CM. Genes that escape from X inactivation. Hum Genet. 2011;130:237-45.

30. Marks H, Chow JC, Denissov S, Francoijs KJ, Brockdorff N, et al. Highresolution analysis of epigenetic changes associated with $\mathrm{X}$ inactivation. Genome Res. 2009;19:1361-73.

31. Rougeulle C, Chaumeil J, Sarma K, Allis CD, Reinberg D, et al. Differential histone H3 Lys-9 and Lys-27 methylation profiles on the X chromosome. Mol Cell Biol. 2004;24:5475-84

32. Mak W, Baxter J, Silva J, Newall AE, Otte AP, et al. Mitotically stable association of polycomb group proteins eed and enx 1 with the inactive $X$ chromosome in trophoblast stem cells. Curr Biol. 2002;12:1016-20.

33. Rugg-Gunn PJ, Cox BJ, Ralston A, Rossant J. Distinct histone modifications in stem cell lines and tissue lineages from the early mouse embryo. Proc Natl Acad Sci USA. 2010;107:10783-90.

34. Deuve JL, Avner P. The coupling of X-chromosome inactivation to pluripotency. Annu Rev Cell Dev Biol. 2011;27:611-29.

35. Ohhata $T$, Senner $C E$, Hemberger M, Wutz A. Lineage-specific function of the noncoding Tsix RNA for Xist repression and $\mathrm{Xi}$ reactivation in mice. Genes Dev. 2011;25:1702-15.

36. Kay GF, Barton SC, Surani MA, Rastan S. Imprinting and X chromosome counting mechanisms determine Xist expression in early mouse development. Cell. 1994;77:639-50.
37. Nesterova TB, Barton SC, Surani MA, Brockdorff N. Loss of Xist imprinting in diploid parthenogenetic preimplantation embryos. Dev Biol. 2001;235:343-50.

38. Kalantry S, Mills KC, Yee D, Otte AP, Panning B, et al. The Polycomb group protein Eed protects the inactive $X$-chromosome from differentiationinduced reactivation. Nat Cell Biol. 2006;8:195-202.

39. Wang J, Mager J, Chen Y, Schneider E, Cross JC, et al. Imprinted X inactivation maintained by a mouse Polycomb group gene. Nat Genet. 2001;28:371-5.

40. Mansour AA, Gafni O, Weinberger L, Zviran A, Ayyash M, et al. The H3K27 demethylase Utx regulates somatic and germ cell epigenetic reprogramming. Nature. 2012;488:409-13.

41. Kamikawa YF, Donohoe ME. Histone demethylation maintains Prdm 14 and Tsix expression and represses xlst in embryonic stem cells. PLoS One. 2015;10:e0125626.

42. Shipony Z, Mukamel Z, Cohen NM, Landan G, Chomsky E, et al. Dynamic and static maintenance of epigenetic memory in pluripotent and somatic cells. Nature. 2014;513:115-9.

43. Eggan $\mathrm{K}$, Akutsu H, Hochedlinger $\mathrm{K}$, Rideout W 3rd, Yanagimachi R, et al. X-Chromosome inactivation in cloned mouse embryos. Science. 2000;290:1578-81.

44. Artus J, Douvaras P, Piliszek A, Isern J, Baron MH, et al. BMP4 signaling directs primitive endoderm-derived XEN cells to an extraembryonic visceral endoderm identity. Dev Biol. 2012;361:245-62.

45. Navarro P, Pichard S, Ciaudo C, Avner P, Rougeulle C. Tsix transcription across the Xist gene alters chromatin conformation without affecting Xist transcription: implications for X-chromosome inactivation. Genes Dev. 2005;19:1474-84.

46. Chaumeil J, Okamoto I, Heard E. X-chromosome inactivation in mouse embryonic stem cells: analysis of histone modifications and transcriptional activity using immunofluorescence and FISH. Methods Enzymol. 2004;376:405-19.

\section{Submit your next manuscript to BioMed Central and we will help you at every step:}

- We accept pre-submission inquiries

- Our selector tool helps you to find the most relevant journal

- We provide round the clock customer support

- Convenient online submission

- Thorough peer review

- Inclusion in PubMed and all major indexing services

- Maximum visibility for your research

Submit your manuscript at www.biomedcentral.com/submit

\section{( ) Biomed Central}

